\title{
Implementação de um modelo de plantio vertical experimental baseado em tecnologia embarcada
}

\author{
Marcos Tiago Araújo de França \\ Universidade Tecnologica Federal do Paraná \\ Medianeira, Brasil \\ tiagitofranca04@gmail.com
}

\author{
Augusto Vaghetti Luchese \\ Universidade Federal do Paraná \\ Palotina, Brasil \\ aluchese@gmail.com
}

\begin{abstract}
This papper presents concepts related to a methodology based on vertical planting, which is framed in a new category known as Smart Farms. Also known as Vertical Farms, this methodology aims to optimize the available planting area, using a column planting system, assisted by advanced irrigation technologies and task automation, the latter benefiting from the advent of IoT. Planting in vertical farms is usually done on an indoor environment, where it is necessary to use alternative lighting sources to sunlight, especially LED lighting, due to the ability to apply specific light spectrum according to the need of culture. Thus, a prototype was developed, based on experiments, in which a culture is planted to assess its viability, where there were problems related to overheating during the execution of routines and the need for adjustments related to the amount of available lighting applied to the crops produced.
\end{abstract}

Resumo- Este trabalho apresenta conceitos referentes à uma metodologia baseada no plantio vertical, que se encontra enquadrada em uma nova categoria conhecida como Smart Farms. Conhecida como Fazendas Verticais, esta metodologia tem como objetivo a otimização da área de plantio disponível, sendo feita a utilização de um sistema de plantio em colunas, assistido por tecnologias de irrigação avançadas e automatização de tarefas, sendo esta última beneficiada com o advento da IoT. O Plantio em modelos verticais geralmente é realizado em um ambiente indoor, onde é necessária a utilização de fontes de iluminação alternativas à luz solar, destacando-se a iluminação LED, devido a capacidade de aplicação de espectros de luz específicos de acordo com a necessidade da cultura. Desta forma, desenvolveu-se, a partir de experimentos, um protótipo, no qual é realizado o plantio de uma cultura para avaliar sua viabilidade onde verificou-se problemas referentes a superaquecimento durante a execução das rotinas e a necessidade de ajustes referentes a quantidade de iluminação disponível aplicada as culturas produzidas.

Palavras-chave-Sistemas Embarcados; IoT; LED; Vertical Farming, Smart Farming.

\section{INTRODUÇÃO}

O agronegócio brasileiro representa aproximadamente $21,6 \%$ do Produto Interno Bruto [1], este resultado é alcançado devido, principalmente, a modernização do campo com a aplicação de tecnologia, resultando ganhos em produção e qualidade. Nessa esteira, surge um novo Estado da Arte aplicado ao agronegócio, conhecido como Smart Farms (do Inglês, Fazendas Inteligentes).

O Sucesso de aplicação das Fazendas Inteligentes está diretamente relacionado a popularização de um novo conceito conhecido como Internet das Coisas (IoT, do Inglês Internet of Things). Para [2], a IoT possibilita a conexão de sensores a objetos de forma que estes, por sua vez, se tornariam inteligentes, possibilitando a tomada de decisões em tempo real através da captura de informações contexto que se está inserido.

No Brasil, o Plano Nacional de Internet das Coisas busca acelerar a implementação da IoT em diversas áreas consideradas prioritárias, como cidades inteligentes, agricultura, saúde e indústria. Estima-se que a implementação deste conceito em larga escala poderia impactar positivamente a agricultura em aproximadamente US\$ 21 bilhões até o ano de 2025 [3].

O modelo das Fazendas Inteligentes, considerando a sua versatilidade de aplicação graças ao uso da IoT, abre possibilidades para a implementação de novas metodologias de plantio em regiões que apresentam problemas referentes ao espaço e/ou acesso à água. Dentre as mais diversas metodologias aplicadas no que tange às Fazendas Inteligentes, destacam-se as Fazendas Verticais (do Inglês, Vertical Farming).

De acordo com [4], as Fazendas Verticais, ou modelos de plantio vertical, são um novo método de plantio que se utiliza de conceitos adotados em sistema de estufas, porém, diferentemente de uma estufa, esta é construída no formato de coluna, possuindo vários andares, de tal forma que como resultado tem-se a otimização de produção em relação a área de plantio.

Graças a aplicação da IoT nas Fazendas Verticais, tem-se como consequência, além da otimização de área útil de produção, o gerenciamento do uso de água considerando a produção sem solo (Hidroponia, Aquaponia ou Aeroponia), em um ambiente controlado (temperatura, umidade do ar, níveis de dióxido de carbono), graças ao monitoramento através do uso de sensores. [5][6].

A aplicação de Fazendas Verticais vem sendo utilizada com sucesso, especialmente no meio urbano, onde criam-se valores sociais, como, por exemplo, a geração de empregos, a otimização de logística e a diminuição do tempo de transporte dos alimentos para o consumidor, permitindo a compra sob demanda, o que diminuiria, ou até mesmo eliminaria o uso de conservantes [5].

Outro fator que garantiu a viabilidade de aplicação dos meios de plantio vertical, foi a popularização de fontes de iluminação alternativas, considerando a impossibilidade da utilização de iluminação solar devido ao ambiente de produção utilizado. Entre os métodos disponíveis a utilização de Diodos Emissores de Luz (do Inglês Light Emitting diodes), também conhecidos como LEDs, pra este fim, é a que 
apresentou melhores resultados, no que tange a substituição da luz natural a um baixo custo energético [4].

A utilização de comprimentos de onda específicos, possibilitado graças ao uso de LEDs, permite ao produtor a obtenção de respostas fotomorfogênicas, bioquímicas ou fisiológicas da cultura produzida, de acordo com suas necessidades [7]. Segundo [8], alguns fótons apresentam-se mais eficientes durante o processo de fotossíntese do que outros, nos quais o espectro de luz Vermelho que varia entre (600 - $700 \mathrm{~nm})$, o azul (400nm - 500nm) são responsáveis por uma melhor taxa de assimilação de $\mathrm{CO} 2$ (produção de $\mathrm{O} 2$ ).

O modelo de plantio vertical proposto por [7], com utilização da combinação de LEDs de cor vermelha e azul em ambiente controlado, obteve uma economia de $90 \%$ de água em relação aos modelos de plantio tradicionais (Figura 1).

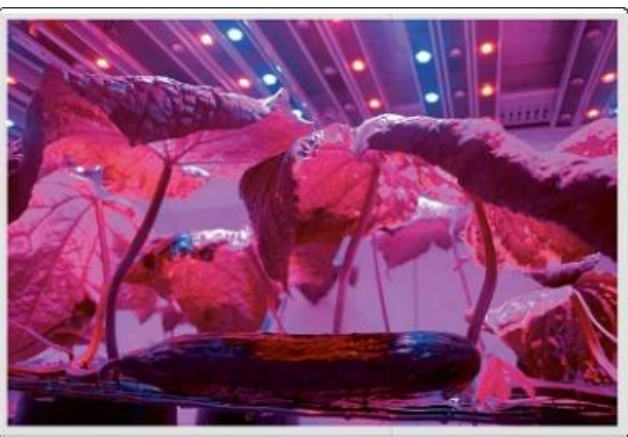

Fig. 1. Unidade de Produção Vertical da PlantLabs dotada de iluminação artificial LED [7]

No Brasil, a aplicação do modelo de plantio vertical é pouco explorada. A Pink Farms foi o primeiro empreendimento da América Latina a utilizá-lo em larga escala, empresa localizada no estado de São Paulo, que surgiu em meados de 2017, e atualmente é centro de referência de plantio vertical para a produção e comercialização de hortaliças folhosas através do uso de iluminação artificial LED (Vermelho + Azul) em conjunto com um sistema de irrigação Hidropônico.

Considerando que no Brasil as Fazendas Verticais são pouco empregadas, visa-se com este trabalho ao desenvolvimento de um modelo experimental de plantio IoT. Com a utilização de ferramentas tecnológicas livres, tais como módulos embarcados para o gerenciamento das unidades de produção, que por sua vez serão dotadas de iluminação LED e de sensoriamento, espera-se o aperfeiçoamento de métricas de variáveis durante o ciclo de vida das culturas.

\section{METODOLOGIA}

O modelo de plantio vertical proposto foi dividido em colunas de produção de 4 níveis, sendo os 3 níveis inferiores responsáveis pela produção de culturas e o nível superior responsável por alocar um reservatório para irrigação e uma a unidade de controle, sendo esta dotada de uma fonte de alimentação e um módulo embarcado para o gerenciamento das tarefas (Figura 2).

As colunas de produção existentes são conectadas entre si utilizando uma topologia mestre e escravo (Figura 3), onde o módulo mestre será responsável por centralizar todos os dados e operações em conjunto com uma Api REST, com o uso de uma conexão serial USB entre os módulos escravo e o mestre.

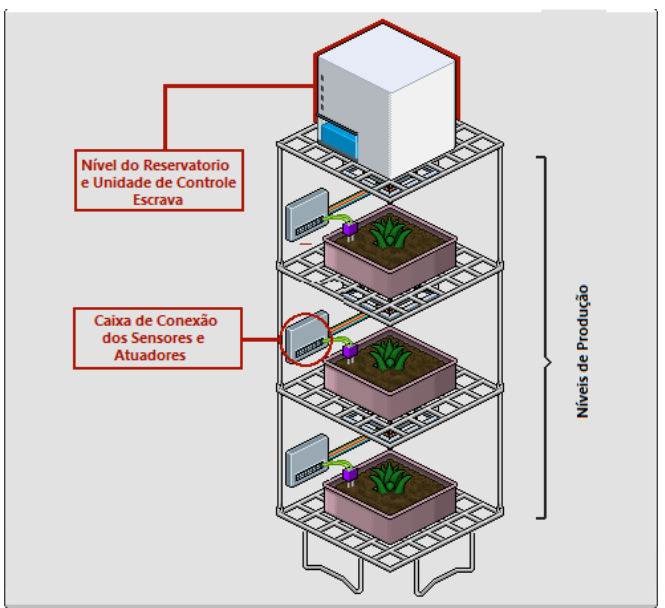

Fig. 2. Projeto Protótipo de uma coluna de produção vertical.

Existem diversos módulos embarcados disponíveis no mercado com características distintas. Desta forma optou-se pela utilização de módulos Arduino Mega 2560, para atuarem como os gerenciadores de nível de produção escravos, considerando seu alto numero de conexões para os sensores e atuadores disponíveis. Para a centralização das operações do modelo vertical como um todo, utilizou-se a plataforma Raspberry PI, considerando o seu poder de processamento superior.

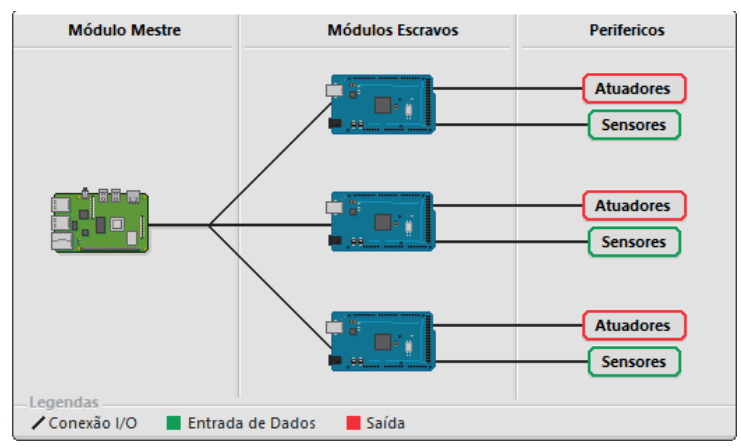

Fig. 3. Topologia Mestre/Escravo utilizada para o gerenciamento das colunas de produção no modelo de plantio vertical.

Cada nível de produção é dotado de uma caixa de conexão (Figura 4a) responsável pela conexão entre os sensores e atuadores presentes no respectivo nível, sendo está uma necessidade considerando que a topologia de construção utilizada prevê o isolamento dos níveis entre si, e à unidade de controle superior (Figura 4b),

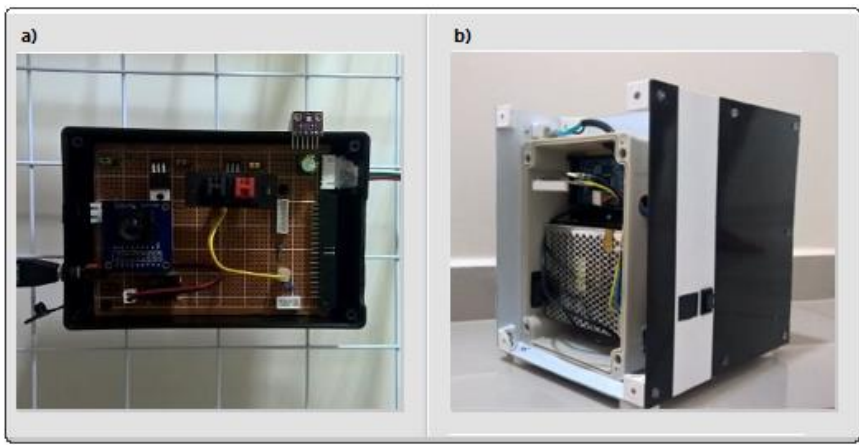

Fig. 4. Módulos Embarcados presentes nas colunas de Produção; a) Caixa de Conexão dos níveis, Caixa de Controle Escrava da coluna. 
Os níveis de produção concebidos são dotados de um conjunto de sensores (Figura 5) que tem como objetivo permitir a coleta de dados ambientais que ajudem na obtenção de dados que possibilitem análises referentes aos fatores que influenciaram o crescimento da amostra no nível de produção, sendo estes: BME280 (Temperatura, Pressão e Umidade do Ar), Módulo Câmera OV7670 (Dados Visuais), MQ135 (Dióxido de Carbono), Modulo de Umidade de Solo.

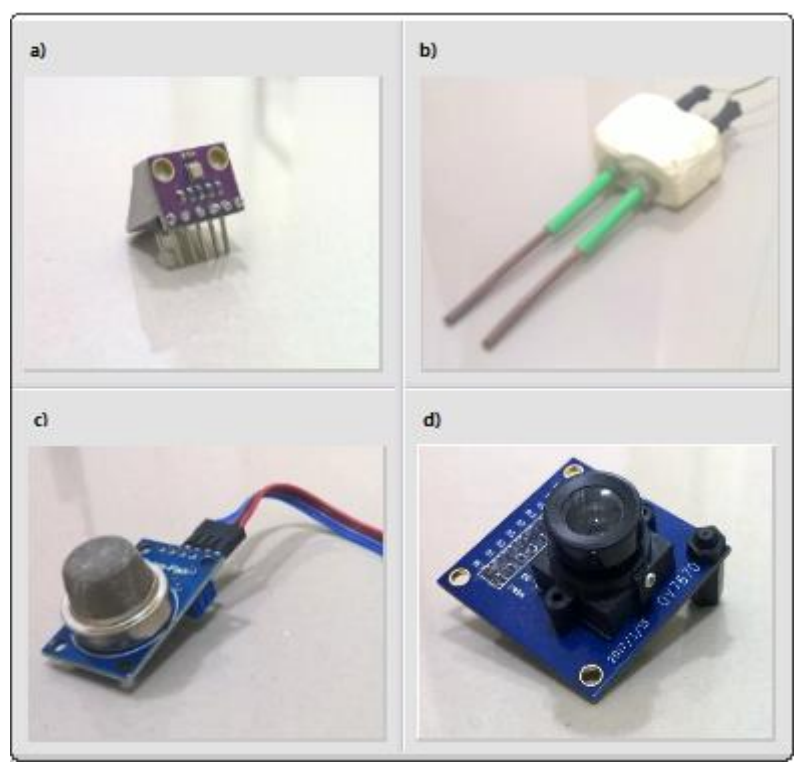

Fig. 5. Conjunto de sensores conectados nas caixas de conexão presentes em cada nível de produção do modelo de plantio vertical; a) Sensor BME280, b) Sensor de Umidade de Solo, c) Sensor MQ-135, d) Câmera OV7670.

Os dados recolhidos a partir destes sensores, com exceção dos dados visuais capturados pelo módulo OPV7670, serão armazenados localmente em cada unidade de controle, além de serem transferidos para a unidade mestre através da conexão Serial USB, podendo assim serem exportados através da API Rest.

Atualmente o sistema de irrigação é composto de vários tanques de $3 \mathrm{~L}$ dotados com sensores de nível. Os tanques possuem condições de adaptabilidade para a técnica de gotejamento. Ressalve-se que esta não é a única alternativa projetada para irrigação, tampouco a mais eficiente, considerando que há também resultados positivos na utilização de Hidroponia, Aquaponia ou Aeroponia.

Desta forma, cada caixa de conexão dos níveis de produção é dotada de conector DC com saída de $3.3 \mathrm{v}$ que permite a utilização de uma hidroponia rudimentar em bandeja (Figura 6), na qual uma solução nutritiva é despejada, e por sua vez é dotada de uma bomba submersa que será conectada a este conector DC e ligada de tempos em tempos, evitando que a solução decante.

Da forma proposta, o modelo de plantio vertical será capaz de lidar com plantio com ou sem solo, com base nas necessidades do experimento. Entretanto, independentemente do tipo de plantio utilizado com base na irrigação, não será possivel a utilização do plantio de sementes, sendo necessária a utilização do transporte de mudas.

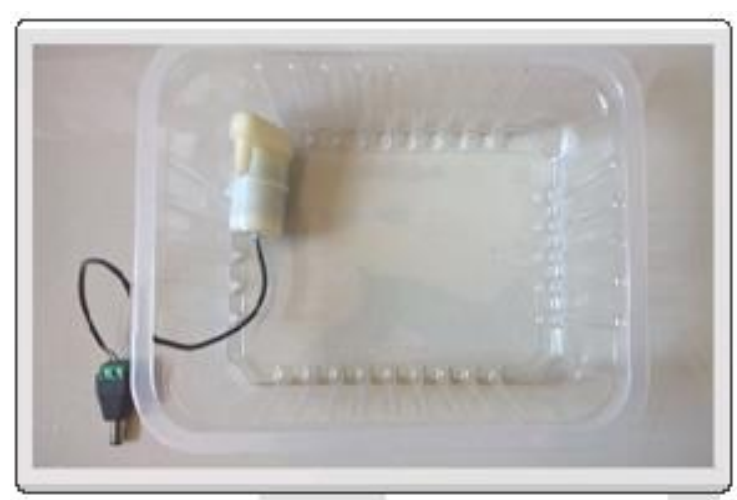

Fig. 6. Bandeja de produção aquapônica dotada de uma bomba submersa.

Sendo o acesso a iluminação o ponto mais importante para a aplicação de um modelo de plantio vertical, optou-se pela a utilização de fitas LED RGB, WS2811 de 12V dotada de capacidade luminosa de 70 lumens $/ \mathrm{m}$, e consumo de $1.1 \mathrm{~W} / \mathrm{m}$ e ângulo de emissão de luz de 120 graus, garantindo o acesso a intensidade necessária e um maior leque de opções para a manipulação do espectro de luz aplicado sobre a cultura.

Com o objetivo de garantir o acesso de iluminação de qualidade, cada nível de produção é dotado de uma estrutura de iluminação dupla quadrada (Figura 7), totalizando 44 módulos de LED RGB (132 LEDs no total), sendo o controle desta estrutura de iluminação realizado pela biblioteca Adafruit NeoPixel.

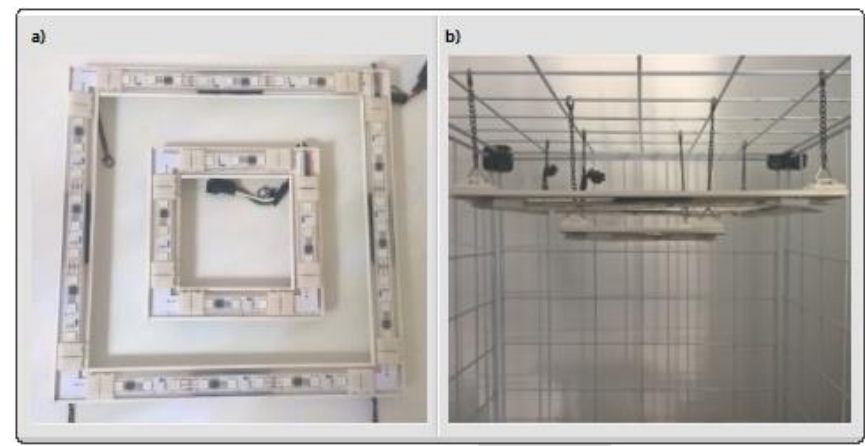

Fig. 7. Estrutura de Iluminação LED presentes nos Níveis de Produção; a) Visão superior do módulo de iluminação, b) Visão Frontal do módulo já instalado no nível de produção.

\section{RESULTADOS PARCIAIS}

Nesta seção serão apresentados os resultados referentes a execução das funções do protótipo de modelo vertical, com base nas especificações apresentadas. Outras analises vão envolver o plantio de uma cultura para validar a quantidade de iluminação disponível para a experimentação.

\section{A. Execução Pratica do Hardware elaborado}

Durante a execução dos primeiros testes de rotina na coluna de produção protótipo (Figura 8), verificou-se que o consumo energético da coluna de produção variou no intervalo de $5.6 \mathrm{~A}$ até $8.2 \mathrm{~A}$, com picos de corrente durante período de iluminação e durante a execução das bombas. Em consequência da alta corrente utilizada, percebeu-se superaquecimento nos conversores de tensão de $5 \mathrm{~V}$ e $3.3 \mathrm{v}$ de cada caixa de conexão presentes nos níveis. 


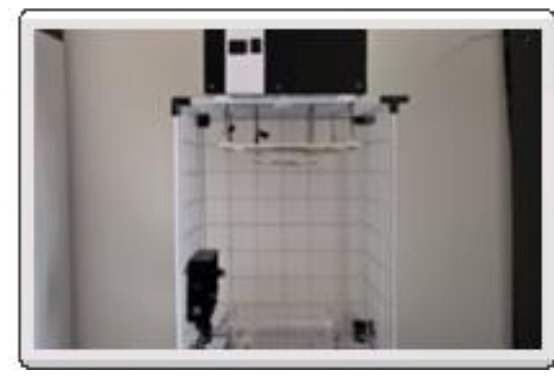

Fig. 8. Protótipo da Coluna de Produção Vertical

\section{B. Experimentação Pratica de Plantio.}

Para o cultivo foi necessária a validação da quantidade de LEDs a ser utilizada, assim foi realizado o transporte de uma cultura de alface crespa (Lactuca sativa var. crispa) em solo (substrato) durante um período de 60 DAT "Dias após o transporte", com o uso de 32 LEDs aplicando a combinação entre o espectro de Luz Vermelho e Azul (Figura 9).

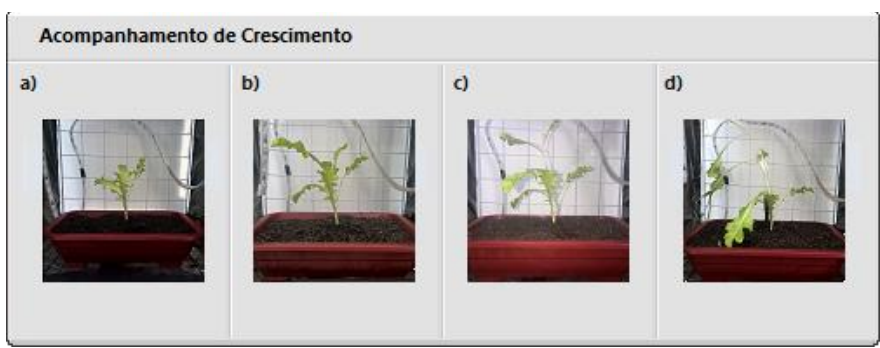

Fig. 9. Experimentação da aplicação de iluminação LED para o crescimento de muda de alface crespa durante um período de 60 dias; a) DAT 0, b) DAT 15; c) DAT 45; d) DAT 60.

Observou-se que cultura no fim da experimentação ficou estiolada, isto é, a quantidade de iluminação presente se mostrou insuficiente, em virtude dessa situação, adicionou-se um segundo suporte de iluminação, complementando a quantidade de LEDs iniciais, totalizando 44 LEDs.

Durante o período de crescimento, foram recolhidos dados referentes ao sensor MQ-135 e BME280, utilizando como parâmetro períodos de hora sinalizados pelo sensor RTC DS3231, onde verificou-se a necessidade da gravação dos dados em intervalos mais curtos, devido a quantidade de memória limitada do módulo Arduino Mega 2560.

\section{PRÓXIMOS PASSOS}

Os próximos passos envolvem a finalização de outras torres de produção (Figura 10), em laboratório, ou seja, isolada do ambiente externo, totalizando 7 colunas (21 níveis de produção), garantindo uma quantidade de amostras que permitirão a execução de experimentos com a aplicação de diferentes espectros de luz sobre a cultura utilizada.

Durante os picos de corrente verificados durante o teste de rotina, verificou-se um aumento significativo na temperatura dos divisores de tensão de 5v (LM7805) e 3.3v (LD1117), assim o uso de dissipadores mostra-se necessário.

Considerando o resultado do primeiro plantio realizado em solo, os futuros experimentos serão realizados sem solo, com uso da bandeja hidropônica, de forma que seja feita a troca da solução de tempos em tempos, considerando que os níveis de produção não contam com um medidor de $\mathrm{Ph}$.

A Captura visual através do modulo OV7670 se mostra inoperante, devido a dificuldades de processamento do módulo escravo Arduino. Entretanto, espera-se conseguir o funcionamento de forma que seja possível a elaboração de um timelapse referente às amostras nos próximos experimentos, abrindo possibilidade para analises por imagem.

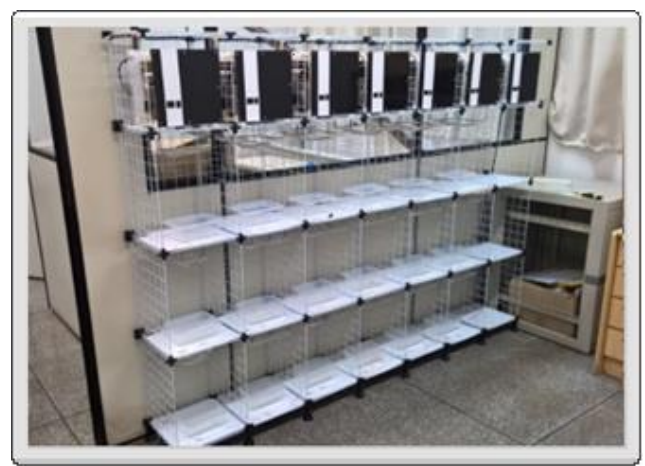

Fig. 10. Estrutura de Plantio vertical proposta em processo de finalização.

Atualmente a API REST construída é rudimentar, permitindo apenas operações de consulta GET de todos os dados capturados, isto é, existe a necessidade de criações de filtros para cada coluna e seus respectivos níveis de produção.

\section{AGRADECIMENTOS}

- Programa de Pós Graduação de Tecnologias computacionais aplicadas ao Agronegócio - PPGTCA UTFPR

- Coordenação de Aperfeiçoamento de Pessoal de Nível Superior- CAPES

\section{REFERÊNCIAS}

[1] MAPA, "Agropecuária Brasileira em Números", 2017, Disponível em <http://www.agricultura.gov.br/assuntos/politicaagricola/agropecuaria-brasileira-em-numeros> [Acesso em: Nov 2020].

[2] Ferreira, P. and Martinho, R. and Domingos, D., "Iotaware business processes for logistics: limitations of current approaches", 2020, Disponível em < http://inforum.org.pt/INForum2014/INForum2010/p apers/internet-das-coisas-e-servicos/Paper099.pdf>.

[3] EMBRAPA, "Internet das Coisas pode ajudar a melhorar produtividade agricola", 2018, Disponível em <https://www.embrapa.br/busca-de-noticias//noticia/31786119/internet-das-coisas-pode-ajudara-melhorar-produtividade-agricola> [Acesso em: Nov 2020].

[4] Despommier, D. "The vertical farm: feeding the world in the 21st century.", New York: Thomas Dunne Books/St. Martin's Press, 2010.

[5] Despommier, D. "Farming up the city: The rise of urban vertical farms", in Trends inbiotechnology, v. 31, p. 388-9, 2013

[6] Zeidler, C. and Schubert, D. and Vrakking, V., "Vertical farm 2.0: Designing an economically feasible vertical farm - a combined european endeavor for sustainable urban agriculture", 2017.

[7] Gomez, C. and Izzo, L., "Increasing efficiency of crop production with leds", in AIMS Agriculture and Food, v. 3, p. 135-153 2018. 